\title{
Correlación de sincronía de la contracción del ventrículo izquierdo con función sisto-diastólica, masa y excentricidad en diversas afecciones cardíacas mediante un software comercial dedicado de SPECT gatillado
}

\author{
Teresa Massardo ${ }^{1}$, Byron Riedel ${ }^{1}$, Enrique Hiplan ${ }^{1}$, Eduardo Swett ${ }^{1}$, Luis Alarcón ${ }^{1}$, Gabriela Paillahueque ${ }^{1}$, Hernán Prat ${ }^{2}$. \\ 1. Sección Medicina Nuclear, Departamento Medicina, Hospital Clínico Universidad de Chile. \\ 2. Departamento Cardiovascular del Hospital Clínico Universidad de Chile. \\ Financiado parcialmente por Proyecto IAEA-CRP E13031
}

Introducción: Actualmente, hay nuevas herramientas de software disponibles para medir la sincronía de la contracción intraventricular izquierda mediante SPECT de perfusión miocárdica. Esta técnica permite identificar anomalías de la conducción, apoyar la terapia de resincronización en insuficiencia cardíaca refractaria e incluso la detección precoz de isquemia.

Objetivo: Conocer la correlación de la sincronía de contracción con otros parámetros de disfunción sisto-diastólica ventricular izquierda.

Método: Estudiamos 135 pacientes remitidos para pesquisa o evaluación de enfermedad coronaria conocida mediante SPECT gatillado. La evaluación de la interpretación inicial con programas QPS/QGS ${ }^{\circledR}$ visual y cuantitativo se efectuó a 50 casos con defectos de perfusión transitoria de diversos tamaños (isquemia), 25 de tipo fijo o mixto (infarto) y 60 sin ellos (normal). Los volúmenes telesistólicos oscilaron entre 26 y $458 \mathrm{~mL}$. Se excluyeron casos con arritmias, anomalías de conducción y artefactos (actividad o movimiento extracardiaco). Los SPECT se procesaron retrospectivamente utilizando el programa Emory Synctool ${ }^{\circledR}$. Del histograma de sincronía de la contracción, el ancho de banda (BW) y la desviación estándar (SD) se correlacionaron con la fracción de eyección (FEVI), volúmenes y excentricidades sistólico / diastólico, masa ventricular izquierda, tasa máxima de llenado (PFR) y tiempo al máximo de llenado (TPFR).

Resultados: Los BW y SD del histograma de fase de contracción fueron mayores en el grupo con defectos fijos y mixtos en comparación con los con perfusión normal. Las correlaciones en reposo y post estrés (Spearman) entre SD y BW con FEVI, volúmenes, excentricidad y masa fueron significativas $(\mathrm{p}<0,0002)$ salvo TPFR que no fue significativa.

Conclusión: La sincronía de contracción intraventricular sistólica izquierda medida con SPECT se correlaciona excelentemente con los parámetros funcionales sistólicos y diastólicos, así como con masa y excentricidad en diversas condiciones y tamaños cardíacos.

Palabras clave: SPECT; perfusión miocárdica; sincronía sistólica. 


\section{Gated Spect evaluation of ventricular systolic synchrony: correlation with ventricular mass and excentricity in different forms of cardiac disease}

New software tools are available to measure left intraventricular contraction synchrony by myocardial perfusion SPECT. This technique allows identification of conduction abnormalities, support resynchronization therapy in refractory heart failure and even allows early detection of myocardial ischemia.

Objective: To determine the correlation of systolic synchrony with other parameters of left ventricular systolic-diastolic dysfunction.

Methods: We studied 135 patients referred for screening or known coronary artery disease evaluation by triggered SPECT. Evaluation of the initial interpretation with visual and quantitative QPS/QGS ${ }^{\circledR}$ programs was performed in 50 patients with transient perfusion defects of various sizes (ischemia), 25 of fixed or mixed type (infarction) and 60 without abnormalities. Telesystolic volumes ranged from 26 to $458 \mathrm{~mL}$. Cases with arrhythmias, conduction abnormalities and artifacts (extracardiac activity or motion) were excluded. SPECT scans were retrospectively processed using the Emory Synctool ${ }^{\circledR}$ software. Histograms of systolic contraction synchrony bandwidth (BW) and standard deviation (SD) were correlated with ejection fraction (LVEF), systolic/diastolic volumes and eccentricities, left ventricular mass, peak filling rate (PFR) and time to maximum filling (TPFR).

Results: $\mathrm{BW}$ and SD of the contraction phase histogram were higher in the fixed and mixed defect group compared to studies showing normal perfusion. Spearman correlations at rest and poststress between SD and BW with LVEF, volumes, eccentricity and mass were all significant $(\mathrm{p}<0.0002)$ except for TPFR.

Conclusion: Left systolic intraventricular contraction synchrony measured with SPECT presents an excellent correlation with systolic and diastolic functional parameters, as well as with mass and eccentricity in various cardiac conditions and ventricular dimensions.

Key words: SPEC, myocardial perfusion, systolic synchronism. 


\section{Introducción:}

Existen diversas entidades patológicas que pueden afectar la función global del ventrículo izquierdo. La sincronía de la contracción se encuentra alterada no solo por las alteraciones de conducción, como bloqueos de rama, sino también por infartos de miocardio, hipertrofia ventricular izquierda secundaria a hipertensión arterial crónica o miocardiopatía de distinto origen (incluyendo la isquémica), casos en que puede existir remodelación patológica. La evaluación de la disfunción sistólica en estas condiciones es compleja e incluye múltiples variables y parámetros, que dependen del método empleado para su pesquisa. Actualmente, la insuficiencia cardíaca con fracción de eyección preservada (IC-FEP) es una entidad de difícil diagnóstico en forma precoz, con pronóstico reservado en algunos casos. Parece importante contar con herramientas que ayuden a su pesquisa inicial pues se pueden presentar como algunas alteraciones de remodelación, disfunción diastólica y eventualmente sistólica. 1,2

La tomografía por emisión de fotón único (SPECT) de perfusión miocárdica gatillada con electrocardiograma está establecida desde hace varias décadas como una herramienta eficiente para evaluar la enfermedad coronaria (EC), tanto en diagnóstico como estratificación de riesgo. ${ }^{3}$ Últimamente, la opción de medir la sincronía mecánica de la contracción intraventricular sistólica del VI, utilizando el análisis de fase basado en las ondas cíclicas de Fourier, entrega información relativamente simple de obtener en un estudio habitual. Posee varias aplicaciones: se ha empleado especialmente en pacientes con bloqueo completo de rama izquierda e insuficiencia cardiaca que no responden a terapia médica optimizada y en candidatos a terapia de resincronización ventricular. Es conocido que existe un porcentaje significativo de casos que no responden a esa terapia y la técnica de análisis de sincronía permitiría seleccionar el último segmento viable que se contrae, para insertar allí el electrodo. Lo anterior es posible con protocolos de esfuerzo mediante ejercicio físico, siendo su utilidad controvertida con vasodilatadores como dipiridamol o adenosina, cuando se utiliza adquisición tardía post-estrés. ${ }^{4}$ Ese protocolo se prefiere para evitar la interferencia de la vasodilatación esplácnica, específicamente por aumento de la actividad hepática ${ }^{5}$.

Dentro de los parámetros que se incluyen en el análisis de fase se encuentran el máximo (peak) del histograma, medido en grados, que representa la fase más frecuente; su desviación estándar (standard deviation: SD) que refleja la distribución de la fase en el histograma; el ancho de banda del histograma (bandwidth: BW) que corresponde a la distribución del 95\% de los elementos de la fase; indica la simetría u oblicuidad (skewness) del histograma, positivo a derecha, $y$, por último, la curtosis que indica el grado de altura del histograma: mientras más alto será más estrecho y mayor será la curtosis. En nuestro centro, contamos con experiencia usando el software Synctool Emory Toolbox ${ }^{\circledR}{ }^{6}$ Participamos en un estudio multicéntrico del Organismo Internacional de Energía Atómica en el que evaluamos pre y post resincronización con sincronía sistólica a 195 pacientes con insuficiencia cardíaca NYHA II-III, refractaria a terapia optimizada con FEVI $\leq 35 \%$,bloqueo completo de rama izquierda y QRS $\geq 120$ ms de 8 centros diferentes ${ }^{7}$. Además, en el grupo de pacientes locales seguimos a 25 casos con evaluación de disfunción endotelial, que mejoraba parcialmente post resincronización ${ }^{8}$.

Tenemos interés en conocer el comportamiento de la sincronía sistólica en nuestros pacientes referidos a SPECT de perfusión en reposo y con dipiridamol post-estrés tardío en distintas condiciones ventriculares izquierdas. Se pretende correlacionar con parámetros funcionales sisto-diastólicas de la curva actividad tiempo que refleja volumen / tiempo ventricular, como también índices de forma y de excentricidad ventricular útiles en insuficiencia cardíaca con remodelación patológica ${ }^{9,10}$. Con técnica isotópica, el índice de excentricidad (IE) utiliza las 3 dimensiones del ventrículo izquierdo y se puede aplicar en imágenes gatilladas o sumadas. En conjunto con la medición de masa ventricular son herramientas cuantitativas del SPECT miocárdico para evaluar remodelación, no utilizadas en la práctica clínica habitual. Así se podría ayudar a pesquisar etapas iniciales del remodelado, como la hipertrofia secundaria a cardiopatía hipertensiva. La dilatación ventricular izquierda se puede acompañar o no de disminución de FEVI, hipertrofia parietal, aumento de masa miocárdica y cambio de forma según el origen y el tiempo de evolución de la condición subyacente. Por otra parte, el ventrículo insuficiente involucra cambios estructurales con múltiples alteraciones a nivel celular y molecular (por ejemplo el factor de necrosis tumoral alfa (TNF- $\alpha$, galectina-3, y Rho-Kinasa entre otros) observadas en un espectro de condiciones de salud como envejecimiento o ejercicio intenso de atletas y otras patologías ${ }^{11}$.

El objetivo de esta investigación preliminar fue correlacionar el histograma de fase de contracción del ventrículo izquierdo con otros parámetros funcionales en pacientes derivados para estudio de patología coronaria con distintos grados de compromiso, evaluados con SPECT 
de perfusión miocárdica.

\section{Material y Método:}

Pacientes: Se estudió retrospectivamente a 135 pacientes consecutivos, 52 hombres $(38.5 \%)$, con promedio de edad de 65 años, derivados al Hospital Clínico de la Universidad de Chile para pesquisa o evaluación de EC con SPECT miocárdico gatillado. En promedio tenían 2 factores de riesgo cardiovascular mayor.

El estudio fue aprobado por el Comité Ético Científico de nuestra Institución para trabajo retrospectivo desde base datos de imágenes PACS y formó parte de una tesis de postgrado (https://repositorio.uchile.cl/bitstream/handle/2250/168546/Patrones-de-sincronismo-ventricular-izquierdo.pdf?sequence $=1 \&$ isAllowed=y).

Criterios técnicos de inclusión: Señal de gatillado ECG estable durante la adquisición; ausencia o grado mínimo de movimiento susceptible de adecuada corrección con software específico; ausencia de actividad significativa del radiofármaco en estructuras extracardíacas que dificulten la delimitación ventricular. También se excluyeron casos con bajo conteo, protocolo de solo 1 día, estrés no farmacológico o que no cumplieran con los criterios de control de calidad del programa Synctool para análisis específico de sincronía.

Protocolo de estrés: Se efectuó prueba de estrés farmacológico con dipiridamol i.v. en dosis alta de $0.84 \mathrm{mg} /$ $\mathrm{kg}$ infundido en 4 min según protocolo del Hospital, con ejercicio isométrico; adquisición de las imágenes de forma tardía, al menos 45 minutos post estrés para evitar hiperemia esplácnica. 5

Técnica Tomográfica SPECT: Se utilizó Tc99mSestamibi y protocolo de dos días en equipo Siemens de doble cabezal, con arco de $180^{\circ}$ en proyección oblicua anterior izquierda y posterior izquierda, dos colimadores de alta resolución y baja energía en $90^{\circ}$; gatillado en ambas fases de estrés y reposo con 8 cuadros. Procesamiento inicial con software Cedars Sinai QPS y QGS $\AA$ visual y cuantitativo, para efectuar reporte original. Se realizó procesamiento retrospectivo con software Synctool de Emory Cardiac Toolbox ${ }^{\circledR}$ de acuerdo con las especificaciones del programa, ajustando la base y ápex del miocardio de forma manual para obtención de parámetros del histograma de sincronía sistólica: BW y SD fueron expresados en grados y parámetros de morfología del histograma: forma y oblicuidad. Del mismo programa se consignaron los parámetros funcionales como fracción de eyección ventricular izquierda (FEVI), volúmenes de fin de diástole (VTD) y sístole (VTS) del ventrículo izquierdo expresados en $\mathrm{mL}$; masa miocárdica expresada en gramos (gr) y tasa máxima de llenado ventricular (PFR) como VTD/seg y tiempo al máximo de llenado (TPFR) expresado en milisegundos (ms) desde la curva de actividad/ tiempo. Se registró adicionalmente el IE sistólico y diastólico, útil para medir remodelación patológica. En este programa el valor 1,0 representa una esfera, en opuesto al programa Cedars Sinai. ${ }^{12}$.

Análisis Estadístico: Se dividieron los casos según el tipo de defecto de perfusión informado en el reporte inicial, comparando los grupos con pruebas t de Student o Mann Whitney; se efectuaron correlaciones entre los parámetros mediante Pearson o Spearman según la normalidad de su distribución.

\section{Resultados:}

La evaluación de la interpretación inicial de los programas Cedars Sinai QPS / QGS ${ }^{\circ}$ en forma visual y cuantitativa se efectuó en 50 pacientes con defectos de perfusión transitoria de diversos tamaños (isquemia), 25 con tipo fijo o mixto (infarto) y 60 sin ellos (normal). De los pacientes con perfusión conservada, el $8 \%(5 / 60)$ tenían función alterada y/o dilatación ventricular izquierda; de los pacientes con algún defecto transitorio el $42 \%$ (21/50) presentaba una función alterada y/o dilatación; de los pacientes con defecto fijo, el 80\% (20/25) tenían función y/o volúmenes alterados.

El electrocardiograma de estrés estuvo alterado en 3,3\% de los pacientes sin defectos de perfusión, en $8 \%$ de los con defecto fijo y en $30 \%$ de los con algún defecto transitorio.

En el análisis de los parámetros funcionales mediante el software Emory la FEVI en reposo varió entre 14\% en paciente con defecto de perfusión miocárdica de tipo mixto y $90 \%$ en uno con defecto transitorio; en post estrés, el rango correspondió a $28 \%$ y $90 \%$. Los volúmenes telesistólicos oscilaron entre $26 \mathrm{~mL}$ en pacientes sin defectos de perfusión y $458 \mathrm{~mL}$ en uno con gran defecto fijo. Los parámetros cuantitativos de isquemia para defectos en estrés (SSS) y para reversibilidad (SDS) variaron entre 0-45 y 0-44 respectivamente. La masa ventricular izquierda varió entre 53 y 345 gr. y la excentricidad en telesístole (IE TS) y telediástole (IE TD) entre 0,04 y 1,0. La Tabla 1 muestra algunos de estos parámetros in-extenso. 
Tabla 1. Medias geométricas y rangos post-estrés y en reposo para los principales parámetros sistólicos: FEVI (\%) y VFD (mL), masa y excentricidad ventricular obtenidos mediante Emory Cardiac Toolbox de acuerdo con los defectos de perfusión. IE TD: índice de excentricidad telediastólico

\begin{tabular}{|c|c|c|c|c|c|c|c|c|}
\hline Defectos & \multicolumn{2}{|c|}{ VTD (mL) } & \multicolumn{2}{|c|}{ FEVI (\%) } & \multicolumn{2}{|c|}{ PFR (VTD/seg) } & \multirow{2}{*}{$\begin{array}{c}\text { Masa (gr) } \\
\text { Reposo }\end{array}$} & \multirow{2}{*}{$\begin{array}{c}\text { IE TD } \\
\text { Reposo }\end{array}$} \\
\hline Perfusión (n) & Post-estrés & Reposo & Post-estrés & Reposo & Post-estrés & Reposo & & \\
\hline \multirow[t]{2}{*}{ Ausente (60) } & 98 & 93 & 82 & 84 & 4,1 & 4,3 & 116,6 & 0,59 \\
\hline & $(35-308)$ & $(26-299)$ & $(28-90)$ & $(25-90)$ & $(2,1-248,8)$ & $(1,8-255,8)$ & (53-233) & $(0,44-0,82)$ \\
\hline Alguno & 111 & 92 & 68 & 72 & 3,4 & 3,5 & 113,2 & 0,7 \\
\hline Transitorio (50) & $(28-276)$ & $(26-255)$ & $(32-90)$ & $(14-90)$ & $(1,5-173,6)$ & $(1,4-8,1)$ & $(61-233)$ & $(0,47-0,94)$ \\
\hline \multirow[t]{2}{*}{ Solo Fijos (25) } & 243 & 206 & 56 & 54 & 3,0 & 3,8 & 187 & 0,68 \\
\hline & $(52-552)$ & (54-458) & $(22-89)$ & $(23-84)$ & $(1,3-76,1)$ & $(1,1-94,1)$ & (88-333) & $(0,49-0,93)$ \\
\hline
\end{tabular}

Los principales parámetros de sincronía sistólica que corresponden a BW y SD fueron en promedio significativamente mayores en el grupo de pacientes con defectos fijos y transitorios en comparación con los estudios de perfusión normal; (Tabla 2).

Las correlaciones significativas en reposo y post estrés entre SD y BW con los distintos parámetros sistólicos y diastólicos evaluados se muestran en la Tabla 3 (post-estrés) y Tabla 4 (reposo). La FEVI, volúmenes telesistólicos y diastólicos, excentricidad y masa ventricular izquierda fueron todas altamente correlacionados con SD y BW ( $\mathrm{p}<0,0001)$. También hubo correlación entre SD y BW de sincronía sistólica con la función diastólica, específicamente el PFR ( $p=0,0002)$; no se encontró correlación con TPFR en ambas fases. La Figura 1 corresponde a gráficos de correlación entre SD y BW del histograma de fase de contracción sistólica con los diversos parámetros funcionales en reposo antes mencionados. En las Figuras 2 y 3 se muestra un par de casos representativos de análisis de fase sistólica.

Por otra parte, en reposo, la masa ventricular izquierda tuvo correlación significativa con los parámetros sistólicos: VTD (r: 0,949 $\mathrm{p}<0,0001$ ), volumen expulsivo (r: $0,830 \mathrm{p}<0,0001)$, VTS ( $\mathrm{r}: 0,796 \mathrm{p}<0,0001)$ y FEVI ( $\mathrm{r}$ : $-0,547 \mathrm{p}<0,0001)$; con los parámetros diastólicos: PFR (r: $-0,474 \mathrm{p}<0,0001)$ y TPFR (r:-0,173 $\mathrm{p}=0.044)$ y con la esfericidad del mismo ventrículo: IE TS (r: 0,621 $\mathrm{p}<0,0001)$ e IE TD (r: 0,284 p=0.0027).

\section{Discusión:}

El SPECT miocárdico gatillado permite un análisis cuantitativo de diversos parámetros funcionales cardiacos.

Tabla 2. Medias geométricas y rangos post-estrés y en reposo para los principales parámetros sistólicos: FEVI (\%) y VFD (mL), masa y excentricidad ventricular obtenidos mediante Emory Cardiac Toolbox de acuerdo con los defectos de perfusión. IE TD: índice de excentricidad telediastólico

\begin{tabular}{|c|c|c|c|c|c|c|c|c|c|}
\hline \multirow{2}{*}{$\begin{array}{l}\text { Defectos } \\
\text { Histograma }\end{array}$} & \multicolumn{3}{|c|}{$\begin{array}{c}\text { Ausente } \\
\text { (n:60) }\end{array}$} & \multicolumn{3}{|c|}{$\begin{array}{l}\text { Cualquier Transitorio } \\
\qquad(n: 50)\end{array}$} & \multicolumn{3}{|c|}{$\begin{array}{c}\text { Solo fijos } \\
\text { (n:25) }\end{array}$} \\
\hline & Post-estrés & Reposo & $p$ & Post-estrés & Reposo & $p$ & Post-estrés & Reposo & $p$ \\
\hline Peak $\left({ }^{\circ}\right)$ & 136 & 132 & ns & 129 & 135 & ns & 95 & 86 & ns \\
\hline $\mathrm{SD}\left({ }^{\circ}\right)$ & $15^{\mathrm{a}, \mathrm{b}}$ & $16^{\mathrm{e}}$ & ns & $23 b 1$ & 8 & ns & $64 a$ & $59 e$ & ns \\
\hline $\mathrm{BW}\left({ }^{\circ}\right)$ & $48^{\mathrm{C}, \mathrm{d}}$ & $46^{f}$ & ns & $64^{d}$ & 46 & ns & $199 c$ & $171 f$ & ns \\
\hline Skewness & 9 & 10 & ns & 9 & 9 & ns & 10 & 10 & ns \\
\hline Curtosis & 110 & 115 & ns & 109 & 105 & ns & 108 & 115 & ns \\
\hline
\end{tabular}




\begin{tabular}{|c|c|c|c|c|c|c|c|c|}
\hline \multicolumn{9}{|l|}{ POST-ESTRÉS } \\
\hline \multicolumn{2}{|c|}{. } & IE TS & IE TD & FEVI (\%) & VTD (ml) & VTS (ml) & Masa (gr) & PFR (VTD/seg) \\
\hline \multirow[t]{2}{*}{ SD $\left({ }^{\circ}\right)$ versus } & r & 0,462 & 0,318 & $-0,580$ & 0,497 & 0,580 & 0,419 & $-0,299$ \\
\hline & $\mathrm{p}$ & $<0,0001$ & 0,0002 & $<0,0001$ & $<0,0001$ & $<0,0001$ & $<0,0001$ & 0,0004 \\
\hline \multirow[t]{2}{*}{ BW $\left(^{\circ}\right)$ versus } & r & 0,441 & 0,268 & $-0,554$ & 0,553 & 0,596 & 0,472 & $-0,317$ \\
\hline & $p$ & $<0,0001$ & 0,0017 & $<0,0001$ & $<0,0001$ & $<0,0001$ & $<0,0001$ & 0,0002 \\
\hline
\end{tabular}

\begin{tabular}{|c|c|c|c|c|c|c|c|c|}
\hline \multicolumn{9}{|l|}{ REPOSO } \\
\hline . & & IE TS & IE TD & FEVI (\%) & VTD (ml) & VTS (ml) & Masa (gr) & PFR (VTD/seg) \\
\hline \multirow[t]{2}{*}{ SD $\left({ }^{\circ}\right)$ versus } & $r$ & 0,539 & 0,426 & $-0,641$ & 0,564 & 0,658 & 0,524 & $-0,399$ \\
\hline & $p$ & $<0,0001$ & $<0,0001$ & $<0,0001$ & $<0,0001$ & $<0,0001$ & $<0,0001$ & $<0,0001$ \\
\hline \multirow[t]{2}{*}{ BW $\left(^{\circ}\right)$ versus } & $r$ & 0,467 & 0,325 & $-0,551$ & 0,617 & 0,635 & 0,566 & $-0,314$ \\
\hline & $p$ & $<0,0001$ & 0,0001 & $<0,0001$ & $<0,0001$ & $<0,0001$ & $<0,0001$ & 0,0002 \\
\hline
\end{tabular}

En este trabajo demostramos que ellos poseen una muy buena correlación con la sincronía ventricular izquierda tanto en reposo como en post-estrés, aunque discretamente mejor en reposo. Nuestros hallazgos demuestran una correlación positiva entre SD y BW con volúmenes telesistólicos y telediastólicos, masa miocárdica y excentricidad ventricular y una correlación inversa con FEVI y PFR, dado que, por ejemplo, a mayor BW (por ende, mayor disincronía) menor fue la FEVI. No observamos cambios significativos de los parámetros de sincronía analizados comparando post-estrés y reposo, lo cual puede estar explicado, como mencionamos anteriormente, por el haber usado test de esfuerzo farmacológico con dipiridamol con adquisición tardía, lo que pudiese enmascarar hallazgos precoces de alteración de la función y sincronía ventricular.

AlJaroudi y cols. en distintos trabajos, han evaluado el impacto de la isquemia en disincronía ventricular izquierda: han observado que en ausencia de defectos de perfusión en SPECT, el histograma de fase de perfusión con tomografía de emisión de positrones (PET) es más angosto en estrés que en reposo, lo que apoya una contracción más eficiente; demostraron que incluso un gran defecto reversible no altera su interpretación; también, por otra parte, que la severidad de la disincronía en casos de disfunción ventricular se asocia a aumento de riesgo de muerte y a descarga apropiada de desfibrilador implantable. Por el contrario, una fase $<50^{\circ}$ se relaciona con ausencia de eventos a 1 año plazo. ${ }^{13}$

Gimelli y cols. ${ }^{14}$. demostraron utilidad para evaluar 


\section{Figura 1.}

Correlación entre los parámetros funcionales en reposo y la sincronía ventricular izquierda en todos los pacientes estudiados.

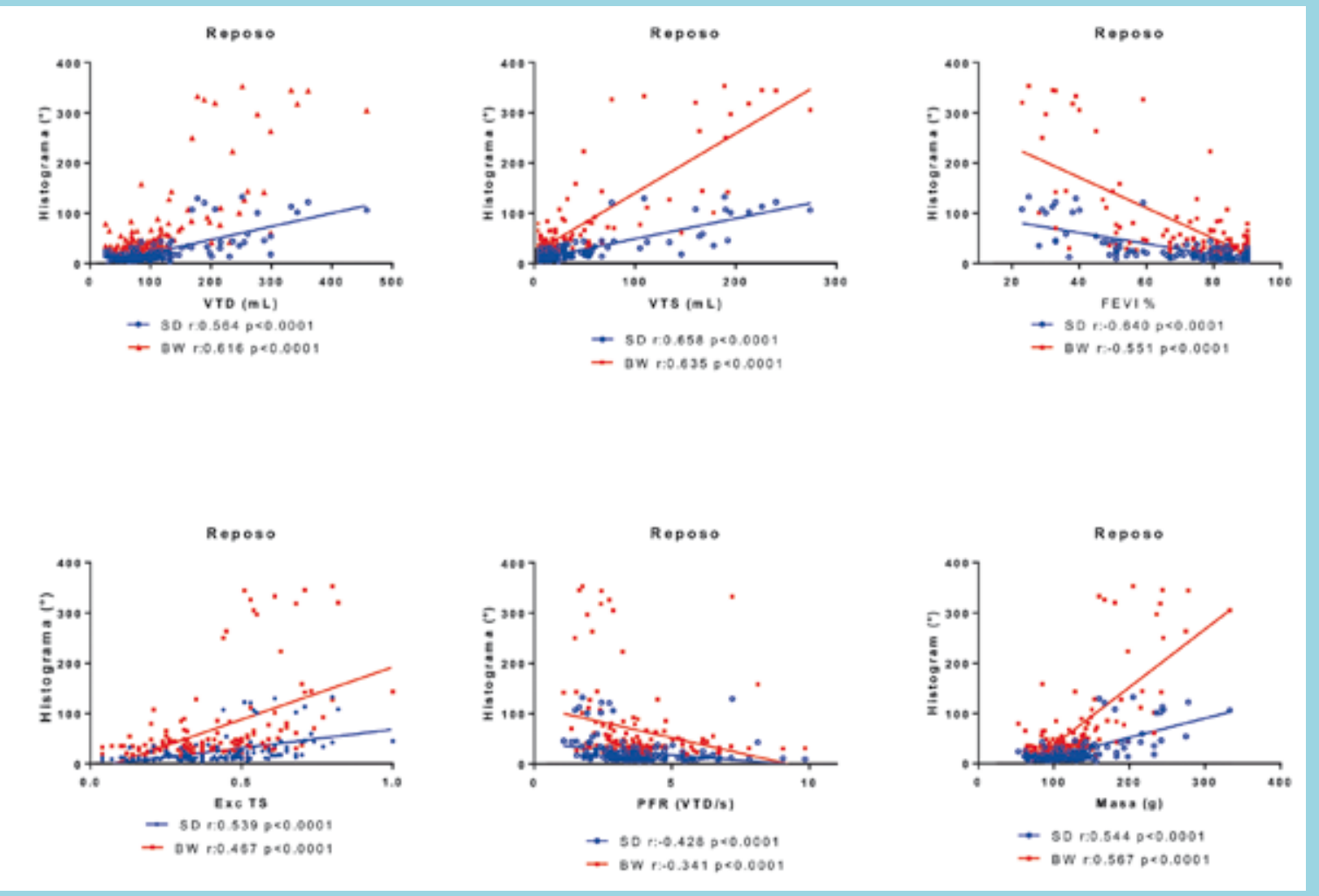

FEVI: Fracción de eyección del ventrículo izquierdo; VFD: volumen diastólico final; SD: desviación estándar del histograma; BW: ancho de banda del histograma; Exc TS: índice de excentricidad en telesístole; PFR: tasa llenado máximo.

isquemia con técnica de sincronía mediante equipo dedicado cadmio zinc teluro (CZT) lo que, según Patel y Mukherjee ${ }^{15}$, también podría tener valor pronóstico; encontraron buena correlación entre las alteraciones de sincronía sistólica de BW y SD con volúmenes/m2 y FEVI, en 657 pacientes (415, de ellos con EC). Del total solo 247 tenían disincronía; esta última fue independiente de la carga de EC según número de vasos comprometidos y de la disfunción sistólica, aunque dependiente de las anormalidades de perfusión y el VTS. Sus valores de correlación a pesar de ser indexados fueron inferiores a los nuestros.

Manejar los parámetros de sincronía y conocer sus alcances tiene importancia clínica. En cuanto a los valores normales, para histograma de fase de sincronía del ventrículo izquierdo, se acepta que la SD media normal es $15,7^{\circ}$. Se ha demostrado que $71 \%$ de los pacientes con insuficiencia cardiaca que cumplen los criterios de resincronización responden al tratamiento si su BW es superior a $135^{\circ} \mathrm{y} / \mathrm{o}$ su $\mathrm{SD}$ es superior a $43^{\circ} 6,16-19$.
Las técnicas de imágenes no invasivas alternativas miden la sincronía sistólica en forma no directamente comparable a la isotópica; la ecocardiografía mediante diferentes técnicas derivadas de 2D, doppler tisular, pulsado y 3D, permite medir sincronía interventricular, intra-ventricular y aurículo-ventricular. Especial utilidad han demostrado recientemente técnicas derivadas del 2D-Speckle-tracking ${ }^{20}$ permitiendo medir strain radial y longitudinal, sincronía intraventricular, trabajo y eficiencia miocárdica mediante curvas presión-strain ${ }^{21}$. Paralelamente, la resonancia magnética cardiaca mediante Feature tracking puede evaluar la alteración mecánica; esta última técnica se ha utilizado para estimar el pronóstico en pacientes con IC-FEp ${ }^{22}$.

Existen diversos estudios que correlacionan parámetros de disfunción ventricular izquierda con valores aumentados de disincronía. Atchley y cols. ${ }^{23}$ demostraron que hasta en un tercio de los pacientes con leve a moderada disfunción se presentaban alteraciones significativas en la sincronía mecánica del ventrículo izquierdo, principal- 


\begin{abstract}
Figura 2 Hombre de 63 años remitido por dolor torácico atípico con un accidente cerebrovascular, hipertensión arterial y diabetes mellitus 2. El mapa polar de perfusión de estrés muestra perfusión reversible de la pared inferior de casi el $50 \%$ de extensión del ventrículo izquierdo, compatible con isquemia. El histograma de fase estrecho y ordenado fue similar en post estrés y reposo, es de tipo normal.
\end{abstract}
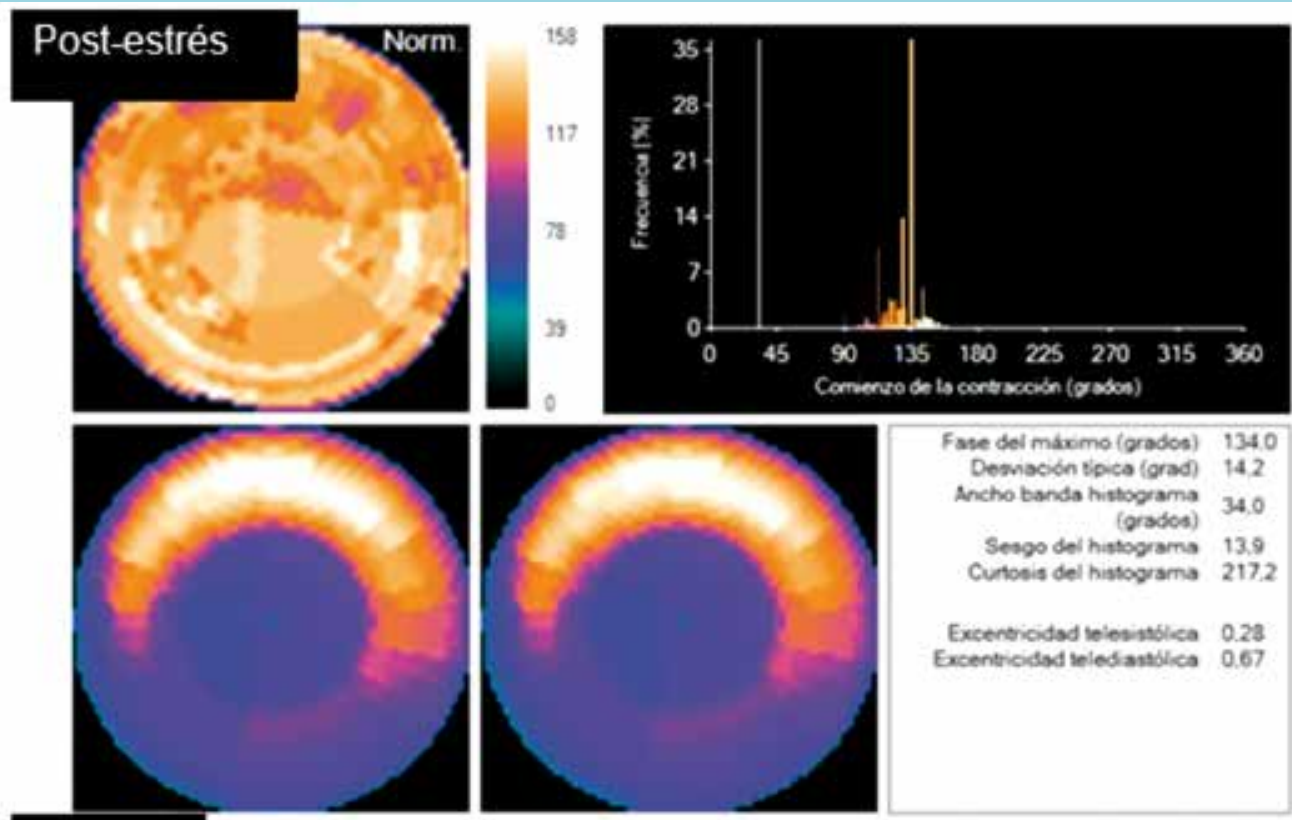

Fase del maxmo (orados) 1340 Desvación tipica (orad) 142 Ancho banda histograma 340 (orados)

Sesgo del histograms 13,9 Curtosis del histograma 217.2

Excentricidad leiesintolea 0.23 Excentricidad ielediantilica 0.67
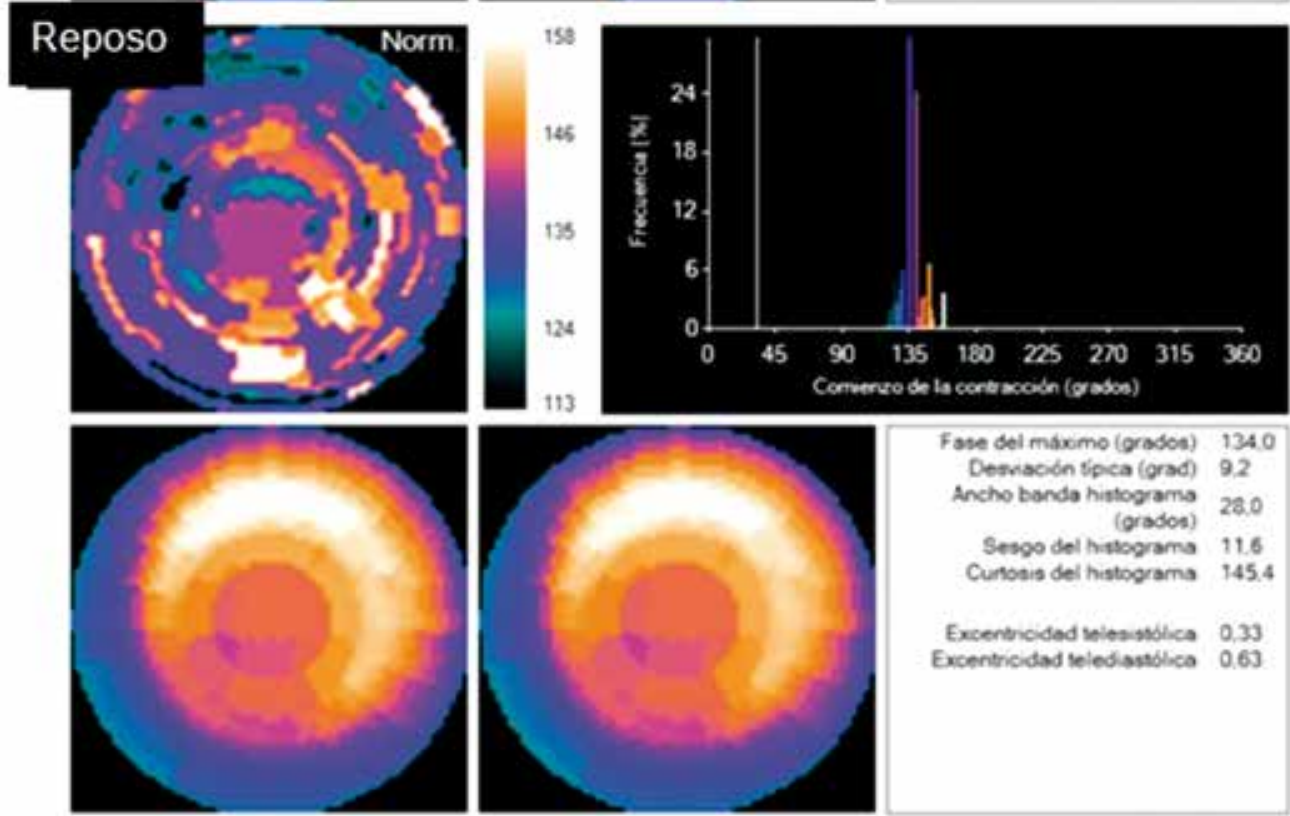

Fase del maximo (grados) 1340 Deswación tipca (orad) 9.2 Ancho banda hintograms 28.0 (grados) 28.0 Sesge del hitbograms 11.6 Cunoais del histograma 145.4

Excentroidad veleastolica 0.33 Excentricidad belediantilica 0.63

mente evidenciados como SD aumentada del histograma de fase. Así mismo, Hessy cols. ${ }^{24}$ demostraron que la disincronía mecánica izquierda reflejada por BW del histograma de fase, medida por SPECT gatillado tiene una mayor asociación con mortalidad, comparada con la medición exclusiva de la sincronía eléctrica por duración del complejo QRS. En pacientes con EC y FEVI $>35 \%$. ambos métodos pueden identificar valores pronósticos desfavorables mejor que la simple FEVI.

Otro punto importante en la evaluación de la función 
Figura 3 Mujer de 56 años con antecedentes de hipertensión arterial esencial e infarto agudo al miocardio previo tratado con stent en arteria descendente anterior, circunfleja y tronco coronario izquierdo. El mapa polar de perfusión muestra defecto mixto anteroseptoapical de $15 \%$ de extensión del ventrículo izquierdo, con reversibilidad de $10 \%$ compatible con isquemia. El histograma de fase muestra BW y SD ensanchados, mayor en estrés comparado con reposo.

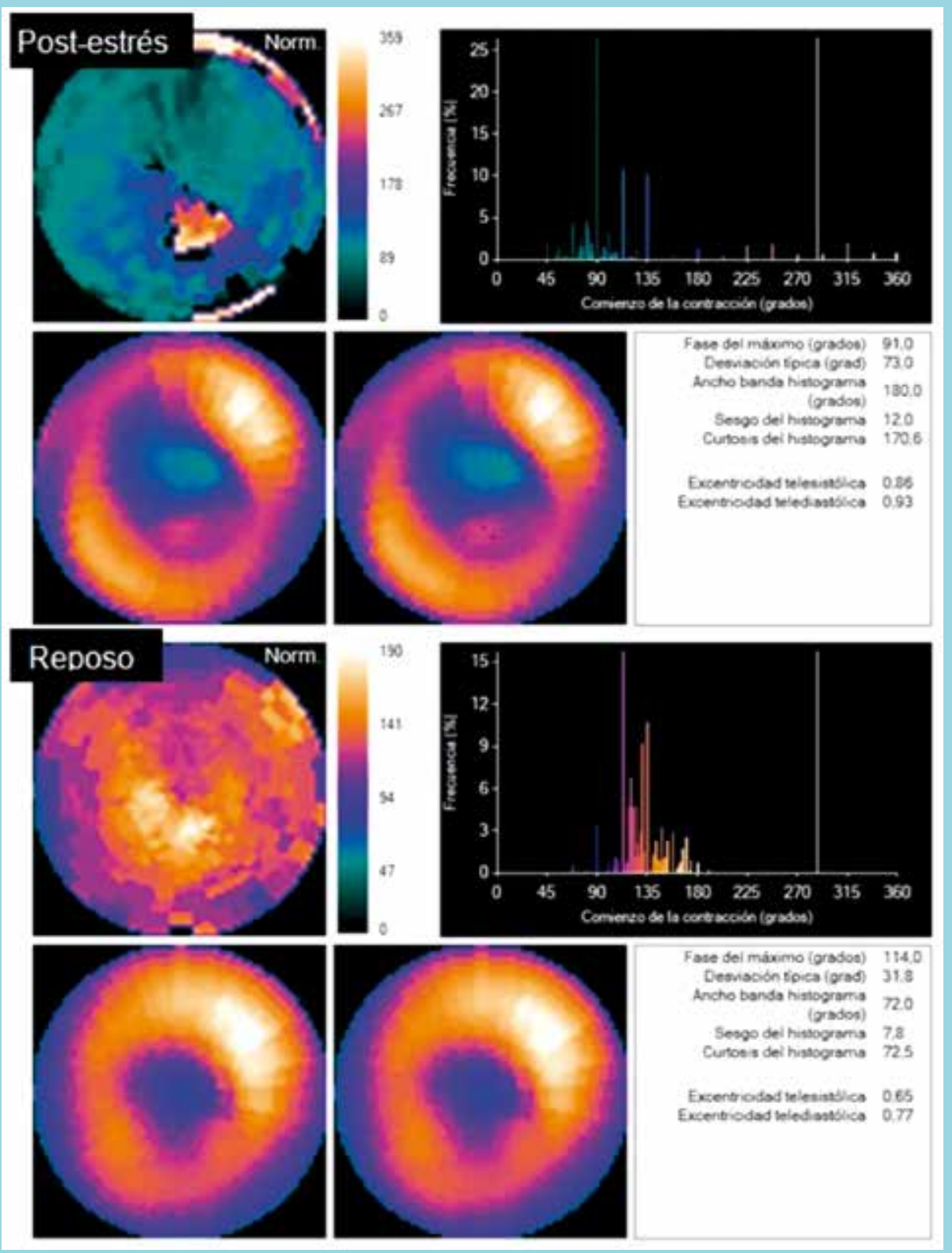

diastólica ventricular izquierda es la medición de la tasa de llenado máximo (PFR). Nitta y cols. ${ }^{25}$ proponen que alteraciones del PFR pueden ser indicadores de isquemia miocárdica, en pacientes con EC no obstructiva, presentando valores bajo el rango normal en EC isquémica posterior al estrés. Gimelli y cols. ${ }^{26}$ también estudiaron las relaciones entre caída de PFR post-estrés y carga isquémica medible en imagen de perfusión miocárdica, en pacientes sin EC obstructiva (lesiones $>20 \%$ y $<50 \%$ en la coronariografía). Así, encontraron que existe una 
correlación significativa entre ambos y permitirían una mejor certeza diagnóstica en detectar EC temprana, comparado con otros parámetros de disfunción sistólica como la caída de FEVI post-estrés. En nuestra serie de datos, encontramos buena correlación inversa del PFR con parámetros de sincronía sistólica como BW y SD.

Finalmente, es bien conocido el papel diagnóstico y pronóstico del SPECT de perfusión miocárdica gatillado con electrocardiograma para detectar áreas de necrosis e isquemia así como sospechar EC multivaso si hay signos indirectos de isquemia balanceada (captación en ventrículo derecho, plétora sanguínea pulmonar, dilatación ventricular post estrés y/o caída de la FEVI con la prueba de provocación ${ }^{27}$, independiente de ausencia de alteraciones de perfusión. Se refuerza así la importancia de la adquisición de las imágenes precoces post-estrés 3, 28, 29 y, como ya se mencionó, mediante el uso del mapa polar de perfusión y el de sincronía, reconocer el último segmento contráctil viable en candidatos a resincronización ${ }^{7}$. A los hallazgos de perfusión miocárdica, se le añaden datos cuantitativos de función y sincronía mecánica del VI que tienen una correlación significativa como evidenciamos en el presente estudio. Ello permite un análisis global más extenso de diferentes parámetros que permitan una mejor interpretación del examen, incluyendo la pesquisa de isquemia ${ }^{30}$.

Estar al tanto de parámetros clínicos adicionales como esfericidad, excentricidad o alteración de la forma ventricular izquierda utilizando SPECT miocárdico, técnica descrita en 2006 por Abidov y cols. ${ }^{31}$, podría permitir reconocer precozmente la remodelación patológica. Existe correlación entre este índice con distintos volúmenes sistólicos y otros parámetros funcionales. En un trabajo previo nuestro de IE con método Cedars se encontró buena correlación, especialmente entre sincronía con VTS. ${ }^{12,} 32$. Por otra parte, el reconocer la disfunción sistólica izquierda se ha demostrado útil en la detección precoz de EC incluso sin alteraciones de perfusión; Gimelli y cols. ${ }^{26}$ reconocieron el valor del PFR, parámetro de disfunción diastólica similar a los nuestros, presentando buena correlación inversa con los parámetros de disincronía sistólica. Por último, como era de esperar, la masa ventricular presenta excelente correlación tanto con los parámetros sistólicos, diastólicos y de esfericidad izquierda, además de los de sincronía de contracción.

El análisis de fase sistólico es reproducible, e incluso se puede aplicar en forma retrospectiva, sin alto costo; requiere controles de calidad habituales a la práctica de medicina nuclear, así como cierto grado de entrenamien- to para la interpretación ${ }^{33}$. Pensamos que si se encuentra disponible debiera ser usado en forma rutinaria, especialmente en casos de insuficiencia cardiaca ${ }^{34}$. Iskandrian, en un editorial reciente, plantea que los parámetros cuantitativos de función y perfusión del SPECT miocárdico aislados deben usarse con cautela y que deben integrarse en el contexto clínico global. ${ }^{35}$.

Actualmente, se cuenta con la opción de cuantificar la sincronía diastólica de forma isotópica, con el mismo software que ha demostrado ser útil en casos de pacientes resincronizados respondedores, en quienes mejoró tanto la disincronía diastólica como sistólica. ${ }^{36}$. Una aplicación importante de ese parámetro es la evaluación de la cardiopatía hipertensiva.

\section{Fortalezas.}

La inclusión fue consecutiva representando la práctica clínica de nuestra población de pacientes referidos a un centro universitario. El análisis de la sincronía fue efectuado por los mismos operadores que no estaban al tanto de la condición clínica de los pacientes y siguiendo estrictamente el control de calidad del proveedor del software.

\section{Limitaciones}

El estudio fue retrospectivo y sin análisis de la coronariografía como parte del mismo. El tamaño de la muestra es limitado; existe mayor número de pacientes sin dilatación ventricular. No hubo disponibilidad de ecocardiografía en todos los casos para una comparación con esta técnica de uso más habitual. No se dispuso de casos sin alteraciones de perfusión ni de función ventricular izquierda indexados por superficie corporal así como tampoco se tabuló el ancho del QRS en reposo, para contar con parámetros normales locales.

\section{Conclusión:}

La evaluación de la sincronía intraventricular sistólica izquierda con SPECT miocárdico gatillado se correlaciona en forma excelente con otros parámetros funcionales sistólicos y diastólicos disponibles, así como con la masa ventricular y excentricidad en diversas condiciones y tamaños cardíacos, tanto en reposo como post-estrés. Consideramos que debiera incluirse en el informe habitual del estudio radio isotópico en estos pacientes.

\section{Declaración de conflicto de interés.}

Los autores no tienen conflicto de intereses con empresas comerciales ni de otro tipo que tengan relación con este trabajo. 


\section{Referencias:}

1. HENNING RJ. Diagnosis and treatment of heart failure with preserved left ventricular ejection fraction. World $\mathrm{J}$ Cardiol. 2020;12(1):7-25.

2. MCDONAGH TA, METRA M, ADAMO M, GARDNER RS, BAUMBACH A, BOHM M, et al. 2021 ESC Guidelines for the diagnosis and treatment of acute and chronic heart failure. Eur Heart J. 2021;42(36):3599-726.

3. MASSARDO T, ALARCON L, SPULER J. Risk stratification of coronary artery disease using radionuclides. Current status of clinical practice. Rev Esp Med Nucl Imagen Mol. 2017;36(6):377-87.

4. SWETT RODRIGUEZ E, HIPLAN, E., ALARCÓN, L., RIEDEL, B., PAILlAHUEQUE, G., PRAT, H., MASSARDO, T. Análisis de sincronismo intraventricular: Ausencia de valor para detección de isquemia en SPECT miocárdico con dipiridamol y adquisición post-estrés tardío. ALASBIMN Journal. 2021 (28 de noviembre).

5. GUTIÉRREZ D, MASSARDO, T, JAIMOVICH, R, ANZOÁTEGUI, W., GUZMÁN, A., LAVADOS, H., ALLIENDE, I. Utilidad del gatillado precoz en SPECT de perfusión miocárdica con tecneciados y vasodilatadores. Problemas inherentes y análisis de experiencia preliminar local. ALASBIMN Journal [Internet]. 2010; 12; 47.

6. CHEN J, GARCIA EV, BAX JJ, ISKANDRIAN AE, BORGES-NETO S, Soman P. SPECT myocardial perfusion imaging for the assessment of left ventricular mechanical dyssynchrony. J Nucl Cardiol. 2011;18(4):685-94.

7. PEIX A, KARTHIKEYAN G, MASSARDO T, KALAIVANI M, PATEL C, PABON LM, et al. Value of intraventricular dyssynchrony assessment by gated-SPECT myocardial perfusion imaging in the management of heart failure patients undergoing cardiac resynchronization therapy (VISION-CRT). J Nucl Cardiol. 2021; 28 (1):55-64.

8. MASSARDO T, PEREIRA, J., SÁEZ, C.G., ARAMBURÚ, I., MORRIS, R., AGUAYO, R., BRUGÈRE, S., PINO, A., HIPLAN, E., PAILLAHUEQUE, G., ALARCÓN. L., SPULER, J., FERNÁNDEZ, R., SWETT, E., SANHUEZA, E., ASENJO, R., PALOMINOS, M.,OLIVARES, N., VALENZUELA, G.,TORRES, J., GARATE. J., KARMELIC, C. Efecto de la terapia de resincronización ventricular en los parámetros de disfunción endotelial y función sistólica izquierda en pacientes con insuficiencia cardíaca crónica y bloqueo completo de rama izquierda. Rev Chil Cardiol. 2018 37(3):11.

9. FIENO DS, HILLENBRAND HB, REHWALD WG, HARRIS KR, DECKER RS, PARKER MA, et al. Infarct resorp- tion, compensatory hypertrophy, and differing patterns of ventricular remodeling following myocardial infarctions of varying size. J Am Coll Cardiol. 2004;43(11):2124-31.

10. DI DONATO M, BARLETTA G, MORI F, FANTINI F. Regional left ventricular wall motion abnormalities in chronic volume overload. Cathet Cardiovasc Diagn. 1983;9(5):453-62.

11. GAASCH WH, ZILE MR. Left ventricular structural remodeling in health and disease: with special emphasis on volume, mass, and geometry. J Am Coll Cardiol. 2011;58(17):1733-40.

12. MASSARDO T, BERROCAL. I., CASTRO. G., MUÑOZ. M., SANTIS, N., PADILLA. P., PRAT. H., ARAYA, V. Valor del índice de excentricidad del ventrículo izquierdo con tomografía spect de perfusión miocárdica en diversas condiciones. Rev Chil Cardiol. 2011; 31: 132-9.

13. ALJAROUDI WA, HAGE FG, HERMANN D, DOPPALAPUDI H, VENKATARAMAN R, HEO J, et al. Relation of left-ventricular dyssynchrony by phase analysis of gated SPECT images and cardiovascular events in patients with implantable cardiac defibrillators. J Nucl Cardiol. 2010;17(3):398-404.

14. GIMELLI A, LIGA R, GIORGETTI A, FAVILLI B, PASANISI EM, MARZULLO P. Determinants of left ventricular mechanical dyssynchrony in patients submitted to myocardial perfusion imaging: A cardiac CZT study. J Nucl Cardiol. 2016;23(4):728-36.

15. PATEL CD, MUKHERJEE A. Assessment of left ventricular mechanical dyssynchrony in coronary artery disease. J Nucl Cardiol. 2016;23(4):737-40.

16. HENNEMAN MM, CHEN J, YPENBURG C, DIBBETS P, BLEEKER GB, BOERSMA E, et al. Phase analysis of gated myocardial perfusion single-photon emission computed tomography compared with tissue Doppler imaging for the assessment of left ventricular dyssynchrony. J Am Coll Cardiol. 2007;49(16):1708-14.

17. CHEN J, GARCIA EV, FOLKS RD, COOKE CD, FABER TL, TAUXE EL, et al. Onset of left ventricular mechanical contraction as determined by phase analysis of ECG-gated myocardial perfusion SPECT imaging: development of a diagnostic tool for assessment of cardiac mechanical dyssynchrony. J Nucl Cardiol. 2005;12(6):687-95.

18. CHEN J, GARCIA EV, LERAKIS S, HENNEMAN MM, BAX JJ, TRIMBLE MA, et al. Left ventricular mechanical dyssynchrony as assessed by phase analysis of ECG-gated SPECT myocardial perfusion imaging. Echocardiography. 
2008;25(10):1186-94.

19. CHEN J, HENNEMAN MM, TRIMBLE MA, BAX JJ, BORGES-NETO S, ISKANDRIAN AE, et al. Assessment of left ventricular mechanical dyssynchrony by phase analysis of ECG-gated SPECT myocardial perfusion imaging. J Nucl Cardiol. 2008;15(1):127-36.

20. NG AC, TRAN DA T, NEWMAN M, ALLMAN C, VIDAIC J, LEUNG DY. Comparison of left ventricular dyssynchrony by two-dimensional speckle tracking versus tissue Doppler imaging in patients with non-ST-elevation myocardial infarction and preserved left ventricular systolic function. Am J Cardiol. 2008;102(9):1146-50.

21. LIU W, HU C, WANG Y, CHENG Y, ZHAO Y, LIU Y, et al. Mechanical Synchrony and Myocardial Work in Heart Failure Patients With Left Bundle Branch Area Pacing and Comparison With Biventricular Pacing. Front Cardiovasc Med. 2021;8:727611.

22. KAMMERLANDER AA, KRAIGER JA, NITSCHE C, DONA C, DUCA F, ZOTTER-TUFARO C, et al. Global Longitudinal Strain by CMR Feature Tracking Is Associated With Outcome in HFPEF. JACC Cardiovasc Imaging. 2019;12(8 Pt 1):1585-7.

23. ATCHLEY AE, TRIMBLE MA, SAMAD Z, SHAW LK, PAGNANELLI R, CHEN J, et al. Use of phase analysis of gated SPECT perfusion imaging to quantify dyssynchrony in patients with mild-to-moderate left ventricular dysfunction. J Nucl Cardiol. 2009;16(6):888-94.

24. HESS PL, SHAW LK, FUDIM M, ISKANDRIAN AE, BORGES-NETO S. The prognostic value of mechanical left ventricular dyssynchrony defined by phase analysis from gated single-photon emission computed tomography myocardial perfusion imaging among patients with coronary heart disease. J Nucl Cardiol. 2017;24(2):482-90.

25. NITTA K, KURISU S, SUMIMOTO Y, IKENAGA H, ISHIBASHI K, FUKUDA Y, et al. Diagnostic value of peak filling rate derived from ECG-gated myocardial perfusion SPECT for detecting myocardial ischaemia in patients with non-obstructive coronary artery disease. Acta Cardiol. 2020;75(1):37-41.

26. GIMELLI A, LIGA R, PASANISI EM, CASAGRANDA M, MARZULLO P. Myocardial ischemia in the absence of obstructive coronary lesion: The role of post-stress diastolic dysfunction in detecting early coronary atherosclerosis. J Nucl Cardiol. 2017;24(5):1542-50.
27. ABIDOV A, GERMANO G, BERMAN DS. Transient ischemic dilation ratio: a universal high-risk diagnostic marker in myocardial perfusion imaging. J Nucl Cardiol. 2007;14(4):497-500.

28. MUT F, GIUBBINI R, VITOLA J, LUSA L, SOBIC-SARANOVIC D, PEIX A, et al. Detection of post-exercise stunning by early gated SPECT myocardial perfusion imaging: results from the IAEA multi-center study. J Nucl Cardiol. 2014;21(6):1168-76.

29. BAJAJ NS, SINGH S, FARAG A, EL-HAJJ S, HEO J, ISKANDRIAN AE, et al. The prognostic value of non-perfusion variables obtained during vasodilator stress myocardial perfusion imaging. J Nucl Cardiol. 2016;23(3):390-413.

30. MASSARDO T. Auxiliary ischemic markers, the role of left ventricular dyssynchrony. J Nucl Cardiol. 2020; 27 (6):2269-72.

31. ABIDOV A, SLOMKA PJ, NISHINA H, HAYES SW, KANG $\mathrm{X}$, YODA S, et al. Left ventricular shape index assessed by gated stress myocardial perfusion SPECT: initial description of a new variable. J Nucl Cardiol. 2006;13(5):652-9.

32. NITTA K, KURISU S, ERASTA R, SUMIMOTO Y, IKENAGA H, ISHIBASHI K, et al. Associations of left ventricular shape with left ventricular volumes and functions assessed by ECG-gated SPECT in patients without significant perfusion abnormality. Heart Vessels. 2020;35 (1):86-91.

33. JIMENEZ-HEFFERNAN A, BUTT S, MESQUITA CT, MASSARDO T, PEIX A, KUMAR A, et al. Technical aspects of gated SPECT MPI assessment of left ventricular dyssynchrony used in the VISION-CRT study. J Nucl Cardiol. 2020.

34. PEIX A, MESQUITA CT, PAEZ D, PEREIRA CC, FELIX R, GUTIERREZ C, et al. Nuclear medicine in the management of patients with heart failure: guidance from an expert panel of the International Atomic Energy Agency (IAEA). Nucl Med Commun. 2014;35(8):818-23.

35. ISKANDRIAN AE. Left Ventricular Perfusion and Function at the Crossroads. J Nucl Cardiol. 2020;27(1):1-2.

36. ALEXANDERSON-ROSAS E, ESPINOLA-ZAVALETA N, GARCIA EV, PEIX A, MASSARDO T, PABON LM, et al. Diastolic dyssynchrony assessment by gated myocardial perfusion-SPECT in subjects who underwent cardiac resynchronization therapy. J Nucl Cardiol. 2021;28 (4):1413-21. 